\title{
Comportamento epidemiológico da malária no Estado de Mato Grosso, 1980-2003
}

\author{
Epidemiological trends of malaria in the State of Mato Grosso, \\ from 1980 to 2003
}

\author{
Marina Atanaka-Santos ${ }^{1}$, Dina Czeresnia ${ }^{2}$, Reinaldo Souza-Santos ${ }^{3}$ \\ e Rosely Magalhães Oliveira ${ }^{3}$
}

\begin{abstract}
RESUMO
Descreveu-se a evolução temporal e espacial de malária em Mato Grosso, discriminadas em períodos de 1980-1985; 1986-1991; 1992-1997 e 1998-2003, distribuídas por microrregião homogênea. O índice parasitário anual do Estado cresceu até 1992, reduzindo para 1,9 casos/mil habitantes em 2003; o coeficiente de mortalidade e a taxa de letalidade foram maiores nos anos de 1980 a 1989. Das 22 microrregiões, 13 apresentaram IPA inferior a 10 casos/1.000 habitantes em todos os períodos, ocorrendo concentração de casos nas microrregiões de Colíder, Alta Floresta, Aripuanã e Alto Guaporé. Em 2003, apenas a microrregião de Aripuanã persistia com IPA superior a 50 casos/1.000 habitantes. As microrregiões de Colíder, em 1983, 1985 a 1988 e 1990 e Alta Floresta, em 1991, apresentaram óbitos acima de 50/100.000 habitantes, sendo a maioria do sexo masculino, na faixa etária de 20 a 49 anos. A distribuição da doença por microrregiões evidenciou que a malária é predominantemente focal.
\end{abstract}

Palavras-chaves: Malária. Distribuição temporal. Análise espacial. Mato Grosso. Epidemiologia.

\begin{abstract}
The temporal and spatial evolution of malaria in Mato Grosso was determined in periods from 1980-1985, 1986-1991, 19921997 and 1998-2003 and distributed by homogeneous microregion. The annual parasitic index of the State rose until 1992 and then diminished to 1.9 cases/1,000 inhabitants in 2003, the ratio of mortality and the lethality rate were greater in the 1980s. Of the 22 microregions, 13 presented an API inferior to 10 cases/1,000 inhabitants in all periods. Cases were concentrated in the microregions of Colíder, Alta Floresta, Aripuanã and Alto Guaporé. In 2003, only the microregion of Aripuanã continued to present an API superior to 50 cases/1,000 inhabitants. The microregions of Colíder, in 1983, 1985 to 1988 and 1990 and Alta Floresta, in 1991 presented deaths over 50/100,000 inhabitants, mainly in males aged 20-49 years. The distribution of the disease in microregions showed that malaria is predominantly found in concentrated sites.
\end{abstract}

Key-words: Malaria. Temporal distribution. Spatial analysis. Mato Grosso. Epidemiology.

No ano 2000, foram notificados 1,14 milhões de casos de malária no Continente Americano, dos quais 53,6\% foram registrados pelo Brasil. De 2,6 milhões de pessoas atendidas em todo o país, em 2000, com suspeita de estar com a doença, confirmaram-se 610.878 casos, correspondendo ao índice de lâmina positiva de 23,9\%. Destes casos confirmados, prevaleceram as infecções por Plasmodium vivax (478.212 lâminas positivas) sobre os $P$. falciparum e mista (131.616 lâminas positivas) ${ }^{11}$. Do total de casos, $99,7 \%$ eram da Região
Amazônica, que apresentou o índice parasitário anual (IPA) de 21,9 casos por mil habitantes. Todavia, entre os estados que compõem a Amazônia Legal, o risco de transmissão mostrou-se variado a exemplo do Estado de Roraima que apresentou o IPA de 110,6 casos por mil habitantes e Mato Grosso de 4,7 casos por mil habitantes, em $2000^{10}$.

0 Estado de Mato Grosso apresentou 15.287 casos correspondendo ao IPA de 16,3 casos por mil habitantes, em $1980^{\circ}$. Este índice apresentou crescimento constante,

\footnotetext{
1.Departamento de Saúde Coletiva do Instituto de Saúde Coletiva da Universidade Federal de Mato Grosso, Cuiabá, MT. 2. Departamento de Epidemiologia da Escola Nacional de Saúde Pública Sérgio Arouca da Fundação Oswaldo Cruz, Rio de Janeiro, RJ. 3. Departamento de Endemias Samuel Pessoa da Escola Nacional de Saúde Pública Sérgio Arouca da Fundação Oswaldo Cruz, Rio de Janeiro, RJ.

Endereço para correspondência: Dra. Marina Atanaka-Santos. Rua 5, Quadra 16, nº 19, Jardim Petrópolis, $78070-060$ Cuiabá, MT, Brasil.

e-mail: slcs@terra.com.br

Recebido para publicação em 11/5/2005

Aceito em 26/1/2006
} 
atingindo o IPA de 95,2 por 1.000 habitantes, em $1992^{\circ}$. A partir deste ano, ocorreu um declínio progressivo do IPA, atingindo em 2002, 0 índice de 2,7 casos registrados por mil habitantes ${ }^{20}$.

Apesar da existência de estudos sobre a malária em Mato Grosso, constatando crescimento e redução de IPA, poucos foram os que buscaram apreender o comportamento da doença numa perspectiva espaço-temporal. Diante do exposto, este trabalho se propõe a efetuar uma análise descritiva da evolução dos casos de malária em Mato Grosso, entre 1980 e 2003, segundo microrregião homogênea de residência.

\section{MATERIAL E MÉTODOS}

0 presente trabalho é um estudo ecológico de enfoque exploratório, pautado em dados secundários. Os dados notificados sobre a malária, entre 1980 e 1997, foram disponibilizados pela Divisão de Estatística da Fundação Nacional de Saúde/Regional Mato Grosso; os dados do período de 1998 e 2002 foram obtidos na Secretaria do Estado de Mato Grosso (SES/MT), armazenados no Sistema de Informações de Malária (SISMAL); os de 2003 foram obtidos no Sistema de Informações de Vigilância Epidemiológica - Malária (SIVEP-Malária), da Secretaria de Vigilância em Saúde do Ministério da Saúde. Os dados censitários e as estimativas populacionais foram obtidos no Instituto Brasileiro de Geografia e Estatística (IBGE) (http://www.ibge.gov.br). Os dados referentes aos óbitos, por local de residência, de 1980 e 2002, foram obtidos no Sistema de Informação sobre Mortalidade (SIM), disponibilizados em CD-ROM pelo Ministério da Saúde. Para os óbitos ocorridos no ano de 2003, os dados foram disponibilizados pela SES/MT, sendo restritas ao número de óbitos por causa e localidade. Os óbitos registrados no período de estudo foram reagrupados tomando como base a CID-09, apenas para assegurar continuidade aos dados anteriores aos de 1995, face às mudanças ocorridas com a implantação da CID-10.

Foram calculados o coeficiente de mortalidade por malária - CMM (total de óbitos por malária dividido pelo total da população no ano), o índice parasitário anual - IPA (total de exames positivos notificados, dividido pelo total da população estimada por microrregião e pelo estado como um todo, multiplicado por mil no ano), índice anual de exame de sangue -IAES (total de exames de sangue realizados, dividido pelo total da população multiplicado por mil no ano), índice de lâminas positivas - ILP (total de lâminas positivas para malária, notificadas no ano, dividido pelo total de lâminas examinadas, multiplicado por cem), índice de lâminas positivas para $P$. falciparum - ILPF (total notificado de lâminas positivas para 0 P falciparum, dividido pelo total de lâminas positivas para malária notificado no ano, multiplicado por cem), índice de lâminas positivas para $P$. vivax - ILPV (total notificado de lâminas positivas para 0 $P$. vivax, dividido pelo total de lâminas positivas para malária notificado no ano, multiplicado por cem), razão $P$. falciparum/ $P$. vivax - F/V (total de lâminas positivas para $P$. falciparum, dividido por total de lâminas positivas para P.vivax) e taxa de letalidade (total notificado de óbitos por malária, dividido pelo total de lâminas positivas, multiplicado por cem no ano). Para o IPA, adotou-se as agregações: a) 0,0 casos/mil habitantes; b) de 0,1 a 9,9; c) 10 a 19,9; d) 20 a 49,9; f) 50 a 79,9; g) 80 a 99,9; h) 100 a 199,9 e; i) 200 a 399,9 casos/mil habitantes.

Como menor unidade espacial utilizou-se a microrregião homogênea (MR) estabelecida pelo IBGE, considerando que, no período estudado, esta sofreu menor oscilação em seus limites territoriais que os municípios. 0 Estado de Mato Grosso, desta forma, apresentava em 2004, 22 microrregiões homogêneas: Aripuanã, Alta Floresta, Colíder, Parecis, Arinos, Alto Teles Pires, Sinop, Paranatinga, Norte Araguaia, Canarana, Médio Araguaia, Alto Guaporé, Tangará da Serra, Jauru, Alto Paraguai, Rosário Oeste, Cuiabá, Alto Pantanal, Primavera do Oeste, Tesouro, Rondonópolis e Alto Araguaia (Figura 1). No período de 1980 a 2003, houve apenas a criação em 1982 da microrregião de Primavera do Oeste.

Para construção dos mapas, recorreu ao artifício de agregar o índice parasitário anual de malária em períodos de seis anos. Os períodos adotados foram: período 1 de 1980 a 1985; período 2 de 1986 a 1991; período 3 de 1992 a 1997 e período 4 de 1998 a 2003. O IPA de cada período foi calculado a partir da média do IPAs anuais, conforme procedimento utilizado por Chaves \& Rodrigues ${ }^{2}$. A organização dos dados e construção dos indicadores foram desenvolvidas no programa Excel. Os mapas foram gerados no programa MapInfo.

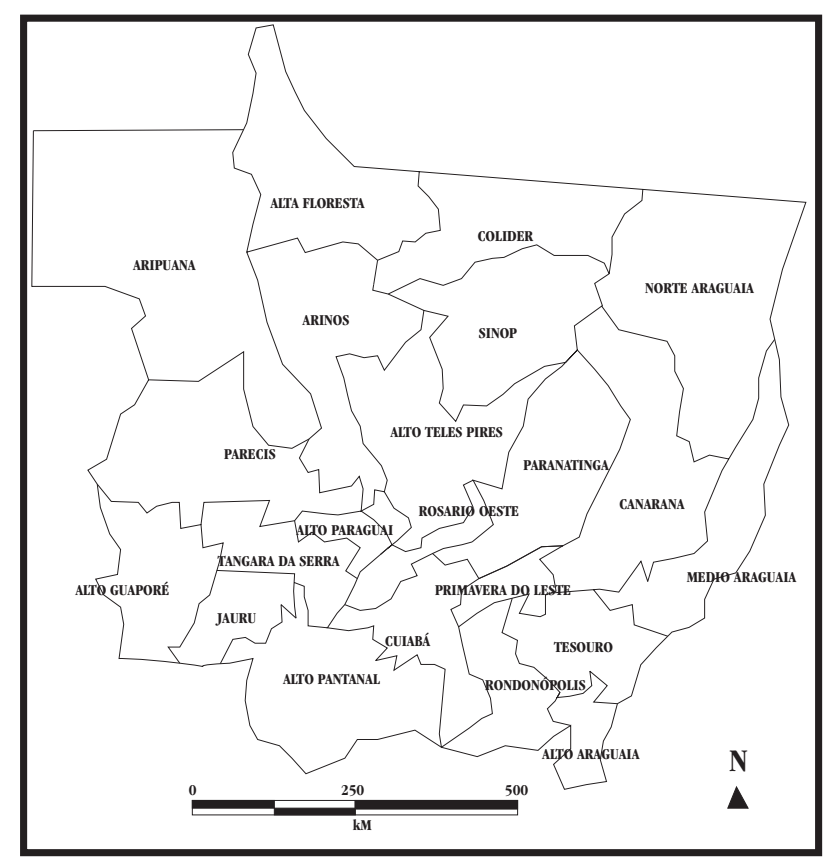

Figura 1 - Microrregiões homogêneas de Mato Grosso, 2004.

\section{RESULTADOS}

A malária em Mato Grosso apresentou tendências distintas entre 1980 e 2003 (Tabela 1). A primeira tendência concentra-se nos anos de 1980 e 1988, no qual ocorreu um gradativo crescimento do IPA, de 11,1 casos/mil habitantes 
Tabela 1 - Distribuição dos índices relacionados à ocorrência de malária, Mato Grosso, 1980 a 2003.

\begin{tabular}{|c|c|c|c|c|c|c|}
\hline Ano & ILP(\%) & ILPF (\%) & ILPV (\%) & IAES (mil) & IPA (mil) & F/V \\
\hline 1980 & 13,9 & 32,3 & 67,1 & 80,0 & 11,1 & 0,5 \\
\hline 1981 & 14,5 & 32,5 & 66,8 & 89,7 & 123,0 & 0,5 \\
\hline 1982 & 14,1 & 41,8 & 58,3 & 81,4 & 11,5 & 0,7 \\
\hline 1983 & 13,3 & 42,2 & 57,5 & 84,2 & 11,2 & 0,7 \\
\hline 1984 & 8,9 & 38,5 & 61,2 & 84,2 & 7,5 & 0,6 \\
\hline 1985 & 9,2 & 41,7 & 58,1 & 90,5 & 8,3 & 0,7 \\
\hline 1986 & 13,9 & 40,2 & 59,5 & 84,7 & 11,8 & 0,7 \\
\hline 1987 & 21,3 & 40,3 & 59,5 & 60,1 & 12,8 & 0,7 \\
\hline 1988 & 21,5 & 40,2 & 59,5 & 72,1 & 15,5 & 0,7 \\
\hline 1989 & 31,9 & 44,1 & 55,7 & 98,7 & 31,5 & 0,8 \\
\hline 1990 & 41,0 & 53,1 & 46,6 & 181,0 & 74,2 & 1,1 \\
\hline 1991 & 39,7 & 48,7 & 50,9 & 180,7 & 71,8 & 1,0 \\
\hline 1992 & 43,9 & 49,1 & 50,4 & 219,0 & 96,1 & 1,0 \\
\hline 1993 & 35,6 & 41,8 & 57,4 & 168,6 & 60,0 & 0,7 \\
\hline 1994 & 30,7 & 39,1 & 60,2 & 143,4 & 44,1 & 0,7 \\
\hline 1995 & 26,2 & 36,1 & 63,2 & 97,5 & 25,5 & 0,6 \\
\hline 1996 & 40,3 & 36,5 & 63,1 & 41,8 & 16,8 & 0,6 \\
\hline 1997 & 19,2 & 31,4 & 68,1 & 36,4 & 7,0 & 0,5 \\
\hline 1998 & 14,6 & 28,9 & 70,2 & 35,1 & 5,1 & 0,4 \\
\hline 1999 & 16,4 & 23,8 & 75,3 & 28,1 & 4,6 & 0,3 \\
\hline 2000 & 22,5 & 21,4 & 78,1 & 20,9 & 4,7 & 0,3 \\
\hline 2001 & 21,7 & 13,4 & 82,5 & 12,3 & 2,7 & 0,2 \\
\hline 2002 & 28,3 & 4,5 & 94,0 & 9,6 & 2,7 & 0,1 \\
\hline 2003 & 32,8 & 11,0 & 87,6 & 5,7 & 1,9 & 0,1 \\
\hline
\end{tabular}

ILP - índice de lâminas positivas; ILPF - índice de lâminas positivas para $P$. falciparum; ILPV - índice de lâminas positivas para $P$. vivax, IAES - índice anual de exames de sangue; IPA - índice parasitário anual; F/N - razão $P$. falciparum / $P$. vivax.

Fonte: Divisão de Estatística FNS/MT (dados de 1980 a 1997); SISMAL/SES-MT (dados de 1998-2002); SIVEP/SVS (dados de 2003).

para 15,5 casos mil/habitantes; a segunda, indica um crescimento mais acentuado do IPA, de 31,5 casos/mil habitantes em 1989 para atingir o índice de 96,1 casos/mil habitantes, em 1992. Esta tendência de crescimento inverte a partir de então, verificando-se uma redução acelerada de 96,1 casos/mil (1992) para 16,8 casos/mil habitantes em 1996. De 1997 a 2003, o declínio é menos acentuado e ocorreu de forma mais gradual, diminuindo de 6,2 casos/ mil habitantes para 1,9 casos/mil habitantes (Tabela 1). 0 IAES acompanha a tendência apresentada pelo IPA, exceto para os anos de 1987 (60,1 exames/mil habitantes) e 1988 (72,1 exames/mil habitantes). Destaca-se no ano de 1992, o registro de 452.002 exames, correspondendo ao IAES de 219 exames/mil habitantes. Deste total de exames notificados, 198.347 lâminas foram positivas para malária, correspondendo ao IPA de 96,1 casos/mil habitantes.

0 índice de lâminas positivas (ILP) foi maior nos anos de 1990, 1992 e 1996 nos quais 42\%, 43,9\% e 40,3\% do total dos exames realizados foram positivos para malária respectivamente. Ocorreu o predomínio de $P$. vivax de 1980 a 2003, exceto no ano de 1990, em que a razão $P$. falciparum/ $P$. $\operatorname{vivax}(\mathrm{F} / \mathrm{V})$ foi de 1,1 lâminas positivas por $P$. falciparum para cada lâmina positiva de $P$. vivax. $O$ índice de lâminas positivas para $P$. falciparum (ILPF) apresentou uma variação ascendente de 1980 a 1990 de 32,35\% para 53,1\% e um decréscimo contínuo, a partir deste ano, reduzindo para 4,5\% em 2002. De 2002 a 2003, ocorreu um aumento de ILPF para $11,0 \%$ (Tabela 1 ).

As microrregiões Alto Teles Pires, Paranatinga, Canarana, Médio Araguaia, Tangará da Serra, Jauru, Alto Paraguai, Rosário Oeste, Alto Pantanal, Primavera do Oeste, Tesouro, Rondonópolis e Alto Araguaia apresentaram IPA inferior a 10 lâminas positivas por mil habitantes em todos os períodos. Os casos concentraram-se na região norte matogrossense (Figura 2). Evidencia a MR Colíder que apresentou no período 2, IPA de 310,0 e no período 3, IPA de 314,7 casos/ mil habitantes, sendo o maior IPA constatado entre as microrregiões e períodos discriminados. As microrregiões Aripuanã e Alta Floresta apresentaram o IPA no período 3 de 155,3 e $209,1 /$ mil habitantes, respectivamente e no período seguinte para 55,6 e 9,6 casos/mil habitantes. A MR Aripuanã destacou-se, no último período, ao permanecer como única microrregião cujo IPA ainda persiste acima de 50 casos/mil habitantes.

Quanto à mortalidade, no período de 1980 a 2003, foram registrados 1.754 óbitos por malária em Mato Grosso, constituindo em $0,9 \%$ dos 198.131 óbitos registrados no estado. Em 1980, o CMM foi de 1,8 óbitos por $100 \mathrm{mil} / \mathrm{habitantes} \mathrm{e}$ atingiu o máximo em 1990 de 9,2 óbitos por 100 mil/habitantes. A partir de então, este coeficiente decresce de forma contínua, chegando a 0,1 óbitos por 100 mil/habitantes, em 2003 (Figura 3). Em 1990, houve maior número de óbitos por malária, coincidindo com o ano em que a razão entre lâminas positivas para $P$. falciparum e $P$. vivax foi de 1,1 casos de $P$. falciparum para um de $P$. vivax (Tabela 1 ). A taxa de letalidade por malária (Figura 3) apresentou um leve declínio entre 1980 e 1981 e teve crescimento entre 1982 e 1985 de $0,1 \%$ para 0,8\%. A partir de 1986, ocorre o declínio gradativo atingindo 0,1\% em 1994. De 1995 a 2001, a taxa de letalidade teve um crescimento de $0,1 \%$ para $0,2 \%$, decrescendo novamente para $0,1 \%$ em 2003.

Os óbitos por malária foram em maior número para os indivíduos do sexo masculino (1.198 óbitos) que feminino (443 óbitos) no período (Tabela 2). Entre os indivíduos do sexo masculino observou-se maior número de óbitos (634) na faixa etária de 20 a 49 anos atingindo 52,9\% do total de óbitos do período, seguido da faixa etária igual ou maior de 50 anos, com 309 (25,8\%) óbitos. Entre o sexo feminino, ocorreu maior número de óbitos (198 óbitos) na faixa etária dos 20 a 49 anos $(44,7 \%)$, seguidos da faixa etária de 0 a 4 anos, com 95 (21,5\%) óbitos.

A distribuição de óbitos por espécie parasitária evidenciou 854 (47,3\%) óbitos causados por malária falciparum, seguida de $747(41,4 \%)$ óbitos por malária não especificada; $145(8 \%)$ por outras malárias, $38(2,1 \%)$ por malária vivax, $22(1,2 \%)$ por malária mista e $1(0,1 \%)$ por malária quartã. $\mathrm{Na}$ distribuição anual dos óbitos por espécie parasitária, observa-se a curva ascendente para aqueles provocados por P. falciparum e para malária não especificada de 1980 a 1990 , e uma redução gradativa de 1991 a 2002. Destaca-se a elevada 


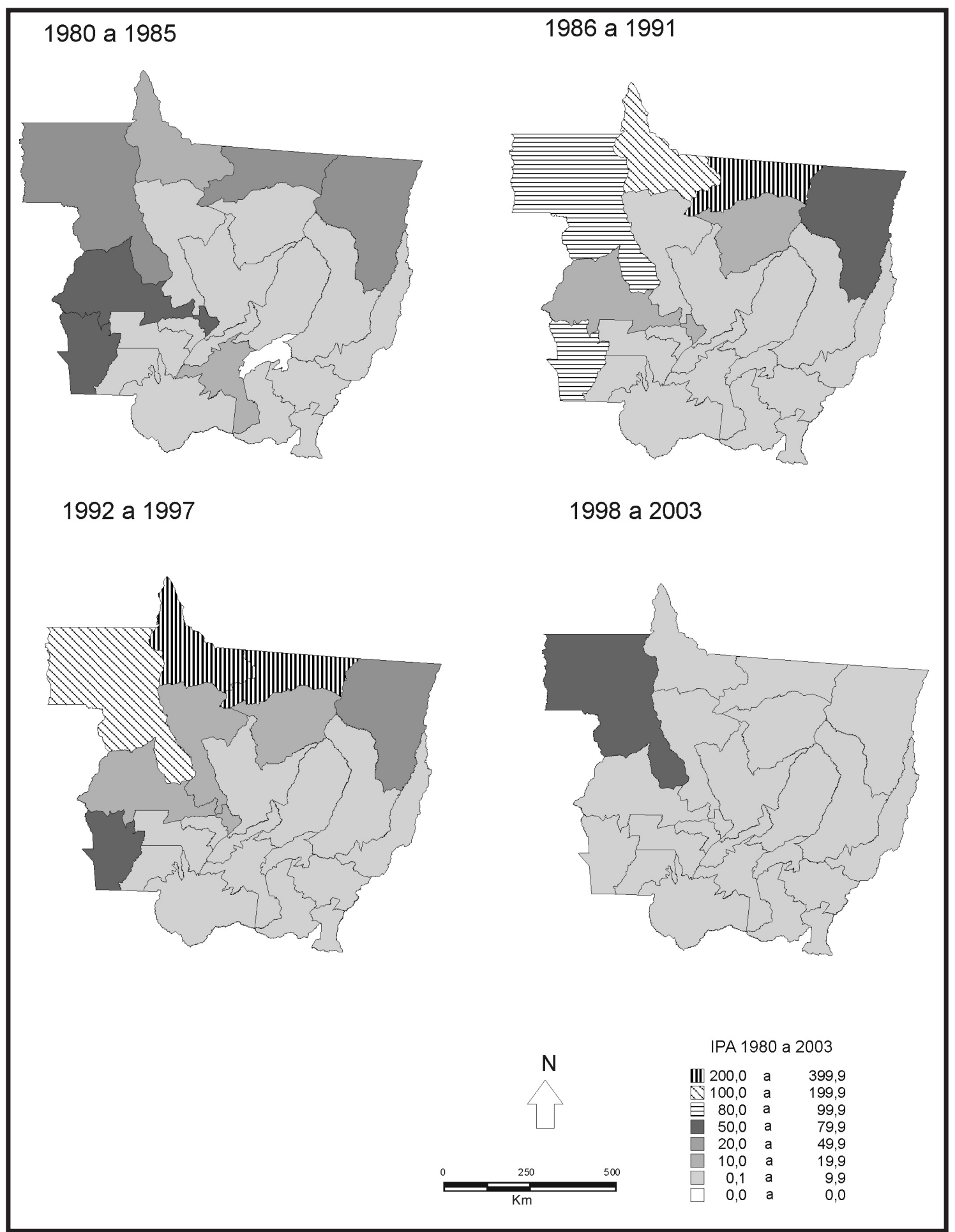

Figura 2 - Distribuição espaço-temporal do índice parasitário anual por microrregião de Mato Grosso, 1980-2003.

Tabela 2 - Óbitos por malária, segundo sexo e faixa etária, Mato Grosso, 1980-2002*.

\begin{tabular}{lcccccr}
\hline Faixa etária & Masculino & \% & Feminino & \% & Total & \% \\
\hline 0 a 4 anos & 119 & 9,9 & 95 & 21,4 & 214 & 13,0 \\
5 a 14 anos & 56 & 4,7 & 59 & 13,3 & 115 & 7,0 \\
15 a 19 anos & 80 & 6,7 & 36 & 8,1 & 116 & 7,1 \\
20 a 49 anos & 634 & 52,9 & 198 & 44,7 & 832 & 50,7 \\
50 anos ou mais & 309 & 25,8 & 55 & 12,4 & 364 & 22,2 \\
\hline Total & $\mathbf{1 . 1 9 8}$ & $\mathbf{1 0 0 , 0}$ & $\mathbf{4 4 3}$ & $\mathbf{1 0 0 , 0}$ & $\mathbf{1 . 6 4 1}$ & $\mathbf{1 0 0 , 0}$
\end{tabular}

Fonte: SIM, 2005

(*) dados disponibilizados até 0 ano 2002.

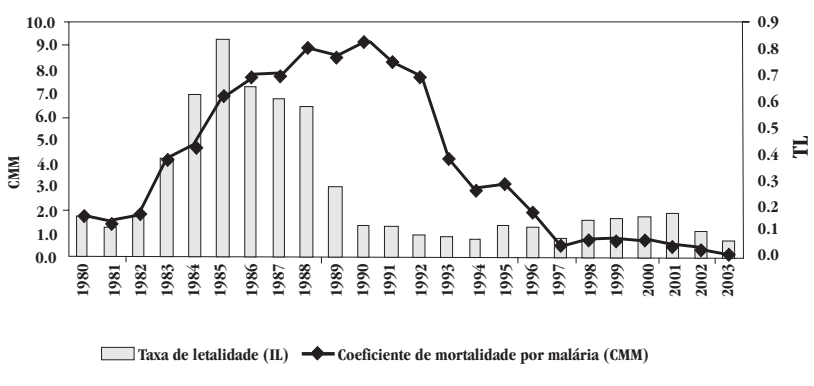

Figura 3 - Tendência do coeficiente de mortalidade por malária (100.000) e taxa de letalidade (\%), Mato Grosso, 1980-2003. 
porcentagem (12 e 40\%) de óbitos cuja a espécie parasitária não foi identificada, ao longo da série.

Os maiores coeficientes de mortalidade por malária ocorreram no período 2 nas microrregiões de Colíder $(53,8$ óbitos por cem mil habitantes), Alta Floresta (33,2/cem mil habitantes) e Aripuanã (22,0/cem mil habitantes). No período 4, o maior coeficiente de mortalidade por malária encontra-se na microrregião de Aripuanã (1,3/cem mil habitantes).

\section{DISCUSSÃo}

0 padrão do IPA apresentado nos anos de 1980 a 2003 (Tabela 1) indica uma situação típica de um processo epidêmico em que ocorrência geralmente é representada no gráfico de tendência como um " $U$ " invertido ${ }^{19}$. Esta tendência crescente de mortalidade, de letalidade e morbidade na década de 1980 foi associada ao aumento do fluxo migratório, mais especificamente à chegada de indivíduos não-imunes trazidos pelos projetos de colonização, procedentes de regiões não endêmicas ${ }^{14}$, em sua maioria, da região sul ${ }^{4}$. Estes eram considerados como migrantes hígidos que, uma vez introduzidos na área malárica, não apresentavam os mecanismos de resistência comum à população tradicional da região. Geralmente, neste grupo populacional a primo-infecção por Plasmodium falciparum apresentava maior letalidade ${ }^{15}$, o que possivelmente imprimiu a tendência ascendente da mortalidade e letalidade na década de 1980. Por outro lado, a incipiente estrutura de serviços de saúde nas áreas de colonização recente, a dificuldade de acesso aos serviços de saúde existente e o difícil acesso dos profissionais de saúde à localidade contribuíram para 0 aumento da mortalidade por malária no estado.

A explosão de casos de malária está associada, no entanto, com a intensificação das atividades garimpeiras na Região Norte Matogrossens ${ }^{16}$ de forma mais expressiva a partir de 1988. A descoberta de ouro aluvião, na região de Peixoto Azevedo, e diamantes em Juína, atraíram os migrantes, inicialmente de garimpeiros nordestinos e nortistas e, mais tarde, dos sulistas que recorreriam a esta atividade como complementar à agricultura ${ }^{4}$. Este agrupamento populacional constituído de garimpeiros era considerado como indivíduos não susceptíveis, uma vez que a exposição freqüente possibilita a aquisição de certa imunidade naturalmente adquirida ${ }^{5}$ e a experiência no trato com a doença assegura certa proteção para a manifestação de malária grave. Esta possibilidade pode ter contribuído para a redução da letalidade, apesar do aumento de lâminas positivas no período 2 (1986-1991). Este aumento de detecção de casos pode ser reflexo da montagem de infra-estrutura de saúde local ou a melhoria no acesso aos serviços de saúde. Outro fator também a ser considerado no aumento da transmissão é a presença de indivíduos assintomáticos em áreas hiperendêmicas 5 .

A partir de 1992, inicia o decréscimo do IPA que foi atribuído pelos profissionais de saúde, entre outros fatores, à intensificação das atividades do programa de controle e a redução da atividade garimpeira na região norte do estado que, possivelmente, provocou uma evasão maciça de uma parcela da população (ou mudança de atividade ocupacional) que viviam em condições e áreas de alto risco de transmissão de malária ${ }^{3}$.

Dentre o total de óbitos registrados no SIM em Mato Grosso, de 1980 a 2003, 0,9\% dos óbitos foi causado por malária, percentual semelhante ao da Amazônia Legal 6 . O CMM e o IPA evoluíram de forma paralela de 1980 a 1990, quando o primeiro começa de decrescer e o segundo continua sua tendência de crescimento até 1992. As microrregiões que apresentaram alta concentração de óbitos foram Alta Floresta, Aripuanã e Colíder, que fazem divisa com os Estados de Rondônia, Amazonas e Pará. Estudo de Pineli cols (1997) ${ }^{13}$, constatou que 40\% dos óbitos por malária registrados em Goiás, no período de 1981 a 1993 eram procedentes de Mato Grosso. Estes óbitos foram constituídos, predominantemente, de indivíduos de sexo masculino e que exerciam, em sua maioria, atividade relacionada ao garimpo. Destaca-se neste estudo a importância das migrações e deslocamentos considerando que, embora os óbitos registrados fossem considerados como de residentes do Estado de Goiás, a última procedência referida era de Mato Grosso.

A ocorrência de maior número de óbitos no sexo masculino, na faixa etária de 20 e 49 anos indica uma exposição predominantemente por atividades laborais. Já a presença de óbitos no sexo feminino e de crianças (Tabela 2) possivelmente está relacionada à transmissão intra e peridomiciliar. Nestes casos, as condições de habitação, de vida e de acesso aos bens sociais e de saúde parecem contribuir significativamente na intensidade da transmissão da doença.

A tendência observada na mortalidade, possivelmente segue o padrão da morbidade semelhante ao que ocorreu em assentamento rural de Rondônia, nos anos de 1991 a 1996. Neste assentamento, mais de $40 \%$ dos casos de malária ocorreu entre os homens na faixa etária de 16 a 40 anos, associada a atividade profissional ${ }^{17}$.

Distintos padrões de transmissão e intensidade são evidenciados ao se destacar as microrregiões por períodos de 1 e 4 (Figura 2). Existem microrregiões de alta incidência como a de Colíder e de baixa incidência como a de Primavera do Oeste. A microrregião de Colíder corresponde à área conhecida como Vale do Rio Peixoto, cuja intersecção da rodovia BR-80 com a BR-163, que liga Cuiabá (MT) a Santarém (PA) era considerada como uma das principais áreas endêmicas para malária no Brasil no início da década de 1990. A elevada incidência nesta localidade era resultante do processo de colonização, combinado à disseminação da atividade garimpeira e condições ambientais favoráveis ao crescimento de vetores ${ }^{21}$. Nestes locais houve intensa exposição devido ao desmatamento, construção de estradas sem drenagem e, especialmente, de habitações precárias $^{22}$. Esta região mostrou-se importante, tanto na produção de casos e óbitos, como na dispersão da doença para o interior do estado e também para outras regiões do Brasil'7.

Esta distribuição heterogênea foi descrita na Amazônia Legal como um comportamento da doença a ser considerado no momento do planejamento das ações de controle ${ }^{141822}$, reafirmando que a malária é uma doença cuja transmissão não se dá de igual intensidade e rapidez em todas as áreas consideradas como malarígenas ${ }^{1}$. 
No entanto, a interpretação dos resultados de estudos realizados a partir de dados secundários requer certa cautela. 0 índice parasitário anual (IPA) é comumente aceito para estimar o risco de ocorrência anual de casos de malária, porém, ao ser construído a partir do número de exames positivos pode não expressar os casos de malária devido ao risco de duplicidade de registro, quando o mesmo paciente for submetido a mais de um exame para verificação de cura, de recrudescências ou recaídas ${ }^{12}$. Esta ressalva deve-se para os dados anteriores à implantação do Sistema de Informações de Malária (SISMAL). Além disso, o registro de mortalidade por malária no SIM pode estar subestimado, principalmente em relação aos óbitos ocorridos na zona rural, especialmente de crianças menores de cinco anos ${ }^{15}$. Neste estudo, o dado de mortalidade de 2003 ficou restrito ao número de óbitos por causa, sendo ainda considerado como preliminar neste ano.

Assim, provavelmente os dados registrados tanto de morbidade como de mortalidade não refletem com precisão a magnitude da epidemia principalmente as que ocorreram no interior dos garimpos e assentamentos. Apesar destas limitações, identificou-se agravamento do quadro epidemiológico da malária em Mato Grosso, na década de 1980 e melhora na década de 1990 e início da década de 2000. A distribuição da mortalidade e morbidade por microrregiões evidenciou a variação no padrão da doença que esteve influenciada por contextos específicos de cada localidade, o que reafirma a concepção de que a malária é uma doença predominantemente focal no Estado de Mato Grosso.

\section{REFERÊNCIAS BIBLIOGRÁFICAS}

1. Barata RB. Malária e seu controle. Hucitec, São Paulo, 1998.

2. Chaves SS, Rodrigues LC. An initial examination of the epidemiology of malaria in the State of Roraima, in the Brazilian Amazon Basin. Revista do Instituto de Medicina Tropical de São Paulo 42:269-275, 2000.

3. Duarte EC, Fontes CJF. Associação entre a produção anual de outro em garimpos e incidência de malária em Mato Grosso - Brasil, 1985-1996. Revista da Sociedade Brasileira de Medicina Tropical 35:665-668, 2002.

4. Ferreira EC. Posse e propriedade territorial: a luta pela terra em Mato Grosso. Editora da Universidade Estadual de Campinas, Campinas, 1986.

5. Fontes CJF. Epidemiologia da malária e fatores associados à infecção assintomática por plasmódio em uma população de garimpeiros da
Amazônia brasileira. Tese de Doutorado, Universidade Federal de Minas Gerais, Belo Horizonte, MG, 2001.

6. Gabriel EMVF. Mortalidade por malária na Amazônia Legal - 1980 a 2000: estudo exploratório. Dissertação de Mestrado, Escola Nacional de Saúde Pública, RJ, 2003.

7. Marques AC. Migration and the dissemination of malaria in Brazil. Memórias do Instituto Oswaldo Cruz 81(supl II):17-30, 1986.

8. Marques AC. Dados epidemiológicos de malária na Amazônia, por município, referente a 1992. Revista da Sociedade Brasileira de Medicina Tropical 26:43-59, 1993.

9. Marques ACE, Pinheiro EA. Fluxos de casos de malária no Brasil em 1980 Revista Brasileira de Malariologia 34:1-31, 1982.

10. Ministério da Saúde. Secretaria de Vigilância em Saúde. Boletim Epidemiológico da Malária $n^{0} 01 / 2003$. SVS, Brasília, 2003. Disponível em : ht tp://dtr2001.saude.gov.br/svs/epi/malaria/pdfs/ be_malaria_01_2003.pdf >. Acesso em: 19/04/2004.

11. Organización Panamericana de La Salud. Informe de la situación de los programas regionales de malaria en las Américas: con base en datos de 2000. Washington, DC, 2001

12. Organização Panamericana de Saúde. Indicadores básicos de saúde no Brasil: conceitos e aplicações. Brasília, Brasil. Disponível: http:// www.opas.org.br/sistema/arquivos/matriz.pdf Acessado em 27/10/2004, 2002.

13. Pineli LL, Araújo ESO, Moraes CA, Netto JCA. Análise epidemiológica de 266 declarações de óbito por malária registradas em Goiás, no período de 1981 a 1993. Revista de Patologia Tropical 26:179-187, 1997.

14. Pinheiro EA. Experiência brasileira na Amazônia. Revista Brasileira de Malariologia e Doenças Tropicais 37:86, 1985.

15. Sabroza PC. Malária no Brasil. Cadernos de Saúde Pública 1:95-99, 1985.

16. Sabroza PC, Kawa H, Campos WSQ. Doenças transmissíveis: ainda um desafio. In. Minayo C (org). Os muitos brasis: saúde e população na década de 1980. Hucitec/Associação Brasileira de Saúde Coletiva, Rio de Janeiro, 1995.

17. Salcedo JMV, Camargo HK, Silva LHP, Camargo LM. Malaria control in na agro-industrial settlement of Rondônia (Western Amazon Region, Brazil). Mem Inst Oswaldo Cruz 95:139-145, 2000.

18. Sawyer DR. Malaria and the environment. Documentos de Trabalho, 13, Brasília, 1992

19. Sawyer DR. Economic and social consequences of malaria in new colonization projects in Brazil. Social Science \& Medicine 37:1131-1136, 1993.

20. Secretaria de Estado de Saúde de Mato Grosso. Boletim Epidemiológico 1:8-9, Mato Grosso, 2004.

21. Soares Filho BS, Sawyer DO. Correlatos ambientais da distribuição espacial da malária em municípios endêmicos no norte do Estado do Mato Grosso. Informe Epidemiológico do SUS 3:47-57, 1996

22. Tauil P. A malária no Brasil. Cadernos de Saúde Pública 1:71-86, 1985. 